\title{
Personalized medicine of type 2 diabetes
}

\author{
Weiping Jia \\ Shanghai Diabetes Institute; Department of Endocrinology and Metabolism, Shanghai Jiao Tong University Affiliated Sixth People's \\ Hospital; Shanghai Key Laboratory of Diabetes Mellitus; Shanghai Clinical Center for Diabetes, Shanghai 200233, China \\ (C) Higher Education Press and Springer-Verlag Berlin Heidelberg 2013
}

Type 2 diabetes is characterized by impaired insulin secretion and decreased insulin sensitivity. It has reached epidemic proportions worldwide and poses a considerable concern for public health. The management of this disease is hence strongly emphasized for the aim of controlling the epidemic. Oral and injectable pharmacologic agents are currently both available for glycemic control. Oral anti-diabetic drugs are among the most widely prescribed, including metformin, sulfonylureas, glinides, a-glucosidase inhibitors, thiazolidinediones and DPP-IV inhibitors [1]. Despite the rapid progress in drug development, it is still challenging to achieve good glycemic control in a substantial population even when multiple anti-diabetic treatments are applied. As is well known, type 2 diabetes is a heterogenous disease, with clinical features, genetic risk factors and underlying pathogenic mechanisms varying among individuals [2]. It is also well recognized that a great inter-individual variability exists in clinical outcomes of hypoglycemic agents. Therefore, poor therapeutic outcomes may be caused by treating patients without concern for the individual characteristics that might influence drug response. Since long-term hyperglycemia is an important contributor of micro- and macro-vascular complications, optimization of treatment strategies according to individual features, the so-called personalized medicine, is imperative.

Response of different individuals to pharmacotherapy may vary due to many factors such as age, gender, liver and/or kidney function and co-medications. It could also be partially attributable to polymorphisms in genes encoding drug-metabolizing enzymes, transporters, receptors and molecules involved in signal transduction. These variants may contribute to the variability in pharmacokinetics (drug absorption, distribution, metabolism and excretion) and pharmacodynamics (drug

Correspondence: wpjia@sjtu.edu.cn target, mechanism of drug action and drug response) of a specific drug, and thus lead to varied efficacious and toxic effects [3]. Studies exploring such inherited differences have updated our knowledge, and the focus has been recently shifted from candidate genes (pharmacogenetics) to genome-wide association studies (pharmacogenomics). Pharmacogenetics/pharmacogenomics approaches are considered to be of importance in the promotion of personalized management of glycemia, helping physicians with the practice of a specific pharmacologic treatment for a genetically defined patient subset, by providing information for the decisions of drug selection, dose titration, treatment duration and avoidance of adverse drug reactions. In addition, they may also shed light on the mechanism of drug action and provide potential therapeutic targets.

Most of the pharmacogenetic studies of type 2 diabetes have focused on oral anti-diabetic drugs including sulfonylureas, glinides, metformin and thiazolidinediones. Numerous genetic markers have been identified so far, particularly those affecting drug disposition (pharmacokinetics). Genetic variants in genes encoding cytochrome P450 (CYP) enzymes contribute substantially to variability in drug efficacy or adverse reaction, as most of the oral hypoglycemic agents are metabolized by these enzymes in the liver. Defective alleles in these genes may be accociated with improved therapeutic effects of hypoglycemic agents and, however, may also be linked to increased incidence of adverse reactions. For example, CYP2C $9 * 2$ and $* 3$ were associated with impaired metabolism and reduced oral clearance of sulfonylureas. Patients carrying these alleles required lower doses of sulfonylureas, and were more likely to achieve glycemic goals including $\mathrm{HbA}_{1 \mathrm{c}}$, but they were at a higher risk of mild or severe hypoglycemia, the major adverse effect of these drugs. In addition, variants in CYP2C8 were found to be associated with efficacy of repaglinide, rosiglitazone and pioglitazone; while polymorphisms in CYP2C9 affected 
nateglinide efficacy. With regard to the process of drug distribution, polymorphisms in genes encoding drug transporters also play important roles in drug efficacy. Variants in the SLCO1B1 gene, which encodes organic anion transporting polypeptide 1B1 (OATP1B1), had impacts on therapeutic effects of repaglinide, nateglinide, rosiglitazone and pioglitazone. Moreover, researchers also found genetic determinants for metformin response in SLC22A1 (encoding organic cation transporter 1) and SLC22A2 (encoding organic cation transporter 2) [4].

Compared with the rapid development in pharmacokinetics, genetic variants influencing pharmacodynamics of hypoglycemic agents are less well characterized. Studies investigating polymorphisms in genes encoding drug targets have led to the identification of some pharmacogenetic markers. KCNJ11 and $A B C C 8$ were found to be harboring genetic markers for sulfonylureas efficacy, and PPAR $\gamma$ harboring genetic variants for thiazolidinediones therapy. Other pharmacodynamic components have also been identified. For instance, variants located in IRS1 and NOS1AP were correlated with sulfonylureas response [4]. To date, more than 60 loci have been identified to confer susceptibility to type 2 diabetes, but the contribution of each locus to disease risk is relatively small. Each diabetes-related locus alone or the combination of all the susceptible loci is thought to have the potential to direct individualized therapy of type 2 diabetes. Therefore, there are attempts to assess the effects of type 2 diabetes-related gene polymorphisms on treatment outcome of anti-diabetic drugs. Such attempts have linked TCF7L2 to sulfonylureas efficacy [4], TCF7L2, KCNJ11, KCNQ1, IGF2BP2, SLC30A8 to therapeutic outcome of repaglinide [5-9], and so on.

Despite the accumulating pharmacogenetic evidence for anti-diabetic medications, pharmacogenomics of these drugs remains in the preliminary stage. As a hypothesis-free approach, genome-wide association study investigates SNPs covering the whole genome, and is regarded as a good biological tool for the identification of genetic markers for treatment outcome. Recently, the first pharmacogenomic study in this field has identified a novel genetic variant (rs11212617), located at a locus containing ATM gene, to be associated with glycemic response to metformin in type 2 diabetes in European populations [10]. This study established the utility of a genome-wide association approach to investigate pharmacogenomics in type 2 diabetes and set an example for future pharmacogenomic research of hypoglycemic therapies. These data also suggest that by such an unbiased way, new biological pathways involved in drug response could be uncovered.

Most of the genetic markers for hypoglycemic agents were identified in populations of European ancestry, whereas the pharmacogenomic advances in Chinese populations are relatively limited. Previous studies have demonstrated the association of CYP2C9, CYP2C19 and $A B C C 8$ with response to sulfonylureas therapy; the association of $P G C-1 \alpha, U C P-2, A C R B 3, S L C 30 A 8$ and $A B C A 1$ with rosiglitazone treatment outcome; the association of $P P A R \gamma, A D I P O Q, L P L$ with pioglitazone efficacy; the association between SLCO1B1 and nateglinide efficacy; the effect of SLC22A2 on pharmacokinetics of metformin in Han Chinese. It is of note that researchers have paid great attention to the pharmacodynamics of repaglinide in patients of Chinese ethnicity in recent years. Numbers of genetic markers were thus found to be associated with repaglinide efficacy, including KCNQ1, NOS1AP, KCNJ11, ABCC8, NAMPT, IGF2BP2, TCF7L2, MDR1, UCP2, SLC30A8, NeuroD1 and PAX4.

Clinical application of pharmacogenomic information has been well established in monogenic diabetes. One of the most stunning examples is the genetic testing for mutations in KCNJ11 in patients with neonatal diabetes. KCNJ11 gene encodes the Kir6.2 subunit of $\mathrm{K}_{\text {ATP }}$ channel in pancreatic $\beta$ cells, and mutations of this gene are present in approximately half of the neonatal diabetes patients. Most of these patients were treated with insulin previously. A great breakthrough occurred when investigators tried to apply sulfonylureas in place of insulin. Effective management of blood glucose concentrations and glycated hemoglobin levels was achieved after transition to sulfonylureas, even after treatment with insulin for many years $[11,12]$.

Unlike those involved in monogenic forms of diabetes, genotypic markers for polygenic diabetes including type 2 diabetes are still far from routine clinical practice. This may be partly due to the following limitations in most of the available data at present. Firstly, the sample size is relatively small, which implies insufficient statistical power of these studies. Secondly, data presented in most of the studies are from healthy volunteers, and the observations established in these studies could thus not be directly implemented to patients with type 2 diabetes. Thirdly, there are no strict definitions for drug response and incidence of adverse drug reactions. All these limitations have hampered the translation of these findings to clinical practice. Prospective clinical trials, demonstrating the usefulness of such testing, are now regarded as strategies to overcome all these limitations. Therefore, need for large, well-designed and wellpowered prospective studies will be highlighted in the future [4]. Moreover, although genotypic markers can serve as complementary tools that can be used together with blood glucose and $\mathrm{HbA}_{1 \mathrm{c}}$ monitoring, algorithms incorporating pharmacogenomic information should be constructed before applying these markers. 
In addition to pharmacogenomic markers, numerous molecular biomarkers including markers derived from proteomics and metabolomics, have been largely investigated in recent years. Proteomics is an approach to examine large numbers of proteins in a body fluid or tissue extract, by which the abundance of proteins and their functional status can be directly assessed. Metabolomics mainly aims at determining metabolite profiles in a specific disease state or for an individual, by measuring a comprehensive set of metabolites in body fluids or tissue extracts. Both approaches may have great potential in providing new insights into pathophysiology of type 2 diabetes and identifying useful biomarkers for personalized medicine of type 2 diabetes. However, proteomic or metabolomic data have not been applied in individualized diabetes management yet. In future, molecular markers identified by these approaches can be used in combination with pharmacogenomic markers to direct treatment decisions. There is also suggestion that epigenetic information should be integrated into personalized management of type 2 diabetes. Whether these new approaches can guide individualized therapy requires substantial further investigation.

\section{References}

1. Ismail-Beigi F. Clinical practice. Glycemic management of type 2 diabetes mellitus. N Engl J Med 2012; 366(14): 1319-1327

2. Smith RJ, Nathan DM, Arslanian SA, Groop L, Rizza RA, Rotter JI. Individualizing therapies in type 2 diabetes mellitus based on patient characteristics: what we know and what we need to know. J Clin Endocrinol Metab 2010; 95(4): 1566-1574

3. Eichelbaum M, Ingelman-Sundberg M, Evans WE. Pharmacogenomics and individualized drug therapy. Annu Rev Med 2006; 57 (1): 119-137

4. Manolopoulos VG, Ragia G, Tavridou A. Pharmacogenomics of oral antidiabetic medications: current data and pharmacoepigenomic perspective. Pharmacogenomics 2011; 12(8): 1161-1191

5. Yu M, Xu XJ, Yin JY, Wu J, Chen X, Gong ZC, Ren HY, Huang Q, Sheng FF, Zhou HH, Liu ZQ. KCNJ11 Lys23Glu and TCF7L2
rs290487(C/T) polymorphisms affect therapeutic efficacy of repaglinide in Chinese patients with type 2 diabetes. Clin Pharmacol Ther 2010; 87(3): 330-335

6. He YY, Zhang R, Shao XY, Hu C, Wang CR, Lu JX, Bao YQ, Jia WP, Xiang KS. Association of KCNJ11 and ABCC8 genetic polymorphisms with response to repaglinide in Chinese diabetic patients. Acta Pharmacol Sin 2008; 29(8): 983-989

7. Yu W, Hu C, Zhang R, Wang C, Qin W, Lu J, Jiang F, Tang S, Bao Y, Xiang K, Jia W. Effects of KCNQ1 polymorphisms on the therapeutic efficacy of oral antidiabetic drugs in Chinese patients with type 2 diabetes. Clin Pharmacol Ther 2011; 89(3): 437-442

8. Huang Q, Yin JY, Dai XP, Pei Q, Dong M, Zhou ZG, Huang X, Yu $\mathrm{M}$, Zhou HH, Liu ZQ. IGF2BP2 variations influence repaglinide response and risk of type 2 diabetes in Chinese population. Acta Pharmacol Sin 2010; 31(6): 709-717

9. Huang Q, Yin JY, Dai XP, Wu J, Chen X, Deng CS, Yu M, Gong ZC, Zhou HH, Liu ZQ. Association analysis of SLC30A8 rs13266634 and rs16889462 polymorphisms with type 2 diabetes mellitus and repaglinide response in Chinese patients. Eur J Clin Pharmacol 2010; 66(12): 1207-1215

10. GoDARTS and UKPDS Diabetes Pharmacogenetics Study Group; Wellcome Trust Case Control Consortium 2, Zhou K, Bellenguez C, Spencer CC, Bennett AJ, Coleman RL, Tavendale R, Hawley SA, Donnelly LA, Schofield C, Groves CJ, Burch L, Carr F, Strange A, Freeman C, Blackwell JM, Bramon E, Brown MA, Casas JP, Corvin A, Craddock N, Deloukas P, Dronov S, Duncanson A, Edkins S, Gray E, Hunt S, Jankowski J, Langford C, Markus HS, Mathew CG, Plomin R, Rautanen A, Sawcer SJ, Samani NJ, Trembath R, Viswanathan AC, Wood NW, Harries LW, Hattersley AT, Doney AS, Colhoun H, Morris AD, Sutherland C, Hardie DG, Peltonen L, McCarthy MI, Holman RR, Palmer CN, Donnelly P, Pearson ER. Common variants near ATM are associated with glycemic response to metformin in type 2 diabetes. Nat Genet 2011; 43(2): 117-120

11. Zung A, Glaser B, Nimri R, Zadik Z. Glibenclamide treatment in permanent neonatal diabetes mellitus due to an activating mutation in Kir6.2. J Clin Endocrinol Metab 2004; 89(11): 5504-5507

12. Pearson ER, Flechtner I, Njølstad PR, Malecki MT, Flanagan SE, Larkin B, Ashcroft FM, Klimes I, Codner E, Iotova V, Slingerland AS, Shield J, Robert JJ, Holst JJ, Clark PM, Ellard S, Søvik O, Polak M, Hattersley AT ; Neonatal Diabetes International Collaborative Group. Switching from insulin to oral sulfonylureas in patients with diabetes due to Kir6.2 mutations. N Engl J Med 2006; 355(5): 467477 